Please do not remove this page

RMIT

UNIVERSITY

\title{
Liquid marble formation: Spreading coefficients or kinetic energy?
}

Eshtiaghi, Nicky; Liu, Jacques

https://researchrepository.rmit.edu.au/esploro/outputs/9921857626801341/filesAndLinks?institution=61RMIT_INST\&index=null

Eshtiaghi, N., \& Liu, J. (2009). Liquid marble formation: Spreading coefficients or kinetic energy? Powder Technology, 196(2), 126-132. https://doi.org/10.1016/j.powtec.2009.07.002

Published Version: https://doi.org/10.1016/j.powtec.2009.07.002

Repository homepage: https://researchrepository.rmit.edu.au

(C) 2009 Elsevier B.V. All rights reserved.

Downloaded On 2023/04/27 01:30:27 +1000

Please do not remove this page 


\section{Accepted Manuscript}

Liquid marble formation: Spreading coefficients or kinetic energy?

Nicky Eshtiaghi, Jacques S. Liu, Wei Shen, Karen P. Hapgood

PII: $\quad$ S0032-5910(09)00410-0

DOI: $\quad$ doi: $10.1016 /$ j.powtec.2009.07.002

Reference: $\quad$ PTEC 7575

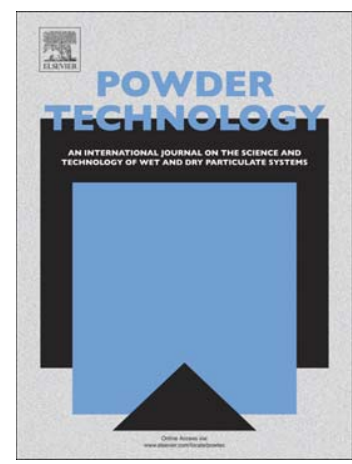

To appear in: $\quad$ Powder Technology

Received date: 12 November 2008

Revised date: 18 June 2009

Accepted date: 13 July 2009

Please cite this article as: Nicky Eshtiaghi, Jacques S. Liu, Wei Shen, Karen P. Hapgood, Liquid marble formation: Spreading coefficients or kinetic energy?, Powder Technology (2009), doi: 10.1016/j.powtec.2009.07.002

This is a PDF file of an unedited manuscript that has been accepted for publication. As a service to our customers we are providing this early version of the manuscript. The manuscript will undergo copyediting, typesetting, and review of the resulting proof before it is published in its final form. Please note that during the production process errors may be discovered which could affect the content, and all legal disclaimers that apply to the journal pertain. 


\section{Liquid marble formation: spreading coefficients or kinetic}

\section{energy?}

\section{Nicky Eshtiaghi ${ }^{a}$, Jacques S. Liua, Wei Shen ${ }^{b}$, Karen P. Hapgood.}

${ }^{a}$ Monash Advanced Particle Engineering Laboratory,

${ }^{\mathrm{b}}$ Australasian Pulp and Paper Institute

Dept. of Chemical Engineering, Monash University, Victoria, Australia, 3800

\section{Abstract:}

A liquid marble is a network of self-assembled hydrophobic powder around a droplet. The mechanism and driving force leading to the formation of liquid marbles has not been investigated. In this study, the solid-liquid spreading coefficient $\left(\lambda_{S L}\right)$ is calculated and the effect of the impact kinetic energy on liquid marble formation for various fluids and particles is investigated. Single drops of fluid were produced using a syringe and released from different heights onto loosely packed powder bed. The degree of powder coverage over liquid droplet after impact was photographed and analysed using image analysis. The results show that the spreading coefficients do not predict liquid marble formation, but instead that powder coverage of the drop is proportional to the applied kinetic energy. As the kinetic energy is increased, the percentage of coverage of liquid droplet by powder increases, and as the particle size decreases the percentage of coverage also increases. These results demonstrate that good powder coverage is assisted by increasing the kinetic energy of impact, which increases the size of the initial fluid-powder contact area and causes internal fluid flow within the droplet during impact and rebound, which entrains the particles and forms the powder shell. The knowledge that the level of agitation applied is an important factor in whether 
liquid marble are successfully produced, is expected to facilitate progress in creating liquid marbles as precursors to a wide range of structured powder-liquid products in cosmetics, pharmaceuticals and other advanced materials.

KEYWORDS: Hydrophobic powder, granulation, nucleation, kinetic energy, liquid marble, powder shell formation, spreading coefficients

\section{Introduction}

Liquid marbles are uniquely structured granules, where a self-assembled network of hydrophobic powder forms a shell around the exterior of a droplet of fluid [1]. Liquid marbles are a novel approach to producing structured fluid-filled particles for in the food, cosmetics and pharmaceutical industries [2, 3, 4].

There are now several papers on the formation of liquid marbles, but there are no studies currently published on why liquid marbles form. .Two different mechanisms have been tentatively proposed to date in the literature:

1. The first suggested mechanism is that liquid marble formation is a surface energy phenomena, driven by the solid-over-liquid spreading coefficient $\left(\lambda_{S L}\right)[2,4]$.

2. The second proposed mechanism is that kinetic energy $[2,3]$ is responsible, based on observations of the role of mixing intensity during the manufacture of a large quantity of liquid marbles.. 
A previous study [2] of liquid marble formation on a loosely packed bed of hydrophobic powder made two critical observations. When a droplet was placed gently onto the powder bed (to avoid fluid flow within the drop interior due to impact or rolling), almost no coverage of the droplet with powder with powder occurred. This implies that the spreading of the powder over the liquid may not be driven by surface tension or spreading coefficients. However, when the drop was released from a height or rolled on the powder surface, an increased tendency to form a complete liquid marble was observed. These preliminary results [2] suggested that bulk motion of the drop due to the kinetic energy of either rolling or impact, is critical for liquid marble formation. This agreed with other observations of liquid marble formation at pilot scale [3], where the use of higher mixer impeller speed to increase the level of agitation and overall kinetic energy applied during mixing was found to be crucial to effectively encapsulating the water in a shell of hydrophobic powder.

In order to form a stable, spherical, liquid marble from a single drop, a series of steps were proposed [2] as part of a framework. The final step in liquid marble formation was provisionally shown as needing a positive value of the solid-over-liquid spreading coefficient $\left(\lambda_{S L}\right)$.The spreading coefficient is defined as the difference between the works of adhesion and cohesion $[5,6]$. Spreading coefficients define that spreading (i.e. further replacement of the liquid -vapor interface with a liquid-solid interface) will occur if it is thermodynamically favourable [6], as indicated by a positive value of the spreading coefficient. Two spreading coefficients are theoretically possible [5] - the liquid may spread over a solid, denoted as $\lambda_{L S}$, or the solid may spread over the liquid, denoted as $\lambda_{S L}$. For liquid marble formation, we are most interested in how a hydrophobic powder covers a liquid droplet, which is theoretically described by $\lambda_{S L}$. 


$$
\lambda_{S L}=-\Delta G_{S L}=\gamma_{S L}+\gamma_{S V}-\gamma_{L V}
$$

A negative value for $\Delta G_{S L}$ and a corresponding positive value for $\lambda_{S L}$ mean the spreading process occurs spontaneously and the solid will freely spread over liquid. Thus, if $\lambda_{S L}>0$ then the solid-liquid interaction is sufficiently strong to promote the spreading of solid over liquid, while if $\lambda_{S L}<0$ then the solid will not spread over liquid.

Spreading coefficients for phase 1 over phase 2 can be calculated from their dispersive component (indicated by superscript d) and polar component (indicated by superscript $p$ ) of the total surface energy (based on Wu's harmonic mean method) $[5,6]$ :

$$
\lambda_{12}=4\left[\frac{\gamma_{1}^{d} \gamma_{2}^{d}}{\gamma_{1}^{d}+\gamma_{2}^{d}}+\frac{\gamma_{1}^{p} \gamma_{2}^{p}}{\gamma_{1}^{p} \gamma_{2}^{p}}-\frac{\gamma_{1}}{2}\right]
$$

Equation (2) can be used to calculate the solid -over-liquid spreading coefficient $\lambda_{S L}$, by defining phase 1 as the solid, and phase 2 as the liquid.

Wu's harmonic mean method is an empirical approach where the forces are divided into polar forces and dispersive forces, and has little fundamental basis. It is intended to be applied only to pure fluids, not to solutions aqueous solutions where adsorption of the polymer at the interface has a significant effect on the local surface energy. Despite this the approach has been widely used to predict solid-liquid spreading interactions, including cases of powders spreading over liquids, with apparent success (for example, see [17] and [18]. In addition, some of these papers, apply the theory to aqueous solutions such as PVP and HPC solutions $[17,18]$ which is theoretically invalid, although again their experimental results appear to 
indicate a causal link between spreading coefficients and granule wetting, strength and/or structure.

The solid-liquid spreading coefficient has been proposed a quantitative method to predict whether a given powder-liquid combination will form a liquid marble [4]. In this paper, we test the hypothesis that the solid-liquid spreading coefficient $\lambda_{S L}$ predicts liquid marble formation, by calculating the $\lambda_{s l}$ spreading coefficient using literature values of surface energy and comparing these results to the corresponding experimental observations of liquid marble coverage whilst holding the kinetic energy as close as possible to zero. The second part of the paper then systematically varies the kinetic energy of the drop at impact and examines the effect on the degree of liquid marble powder coverage, as well as the effect of varying powder size and liquid properties.

\section{Experimental}

\subsection{Spreading coefficient experiments}

For the spreading coefficient experiment, we used $100 \mu \mathrm{m}$ Polytetrafluoroethylene (PTFE) spheres with droplets of different fluids: distilled water, glycerol (99\%, Sigma Aldrich Riedel-de Haen), 6\% w/w polyvinylpyrrolidone (PVP(40T), Sigma Aldrich Pty Ltd) and $2 \% \mathrm{w} / \mathrm{w}$ hydroxypropylmethylcellulose (HPMC, Premium LV, E3, The DOW Chemical Company). Each droplet was released from either $0 \mathrm{~cm}$ or $10 \mathrm{~cm}$ onto a powder bed using $1 \mathrm{cc}$ syringe. PVP and HPMC solution viscosities were measured using a rotational viscometer (Visco Basic Plus, Fungilab, Barcelona). 


\subsection{Kinetic energy experiments}

In order to investigate powder motion mechanism, kinetic energy experiments consisted of two phases - firstly using liquids with different viscosity and secondly using powders with different particle size. A loosely packed powder bed of PTFE 100 (Sigma Aldrich Pty Ltd.) was prepared by sifting the powder through a coarse sieve and collecting the powder in a petri dish. The powder surface was levelled by gently scraping the powder surface with a flat edge. The resulting powder bed was $1.2 \mathrm{~cm}$ high (level with the top of the petri dish) and $61 \%$ porosity. Droplets were dispensed from a $1 \mathrm{cc}$ syringe using an $18 \mathrm{~g}$ needle with $0.02 \mathrm{~mL}$ volume. The syringe needle was oriented horizontally, parallel to the powder bed so that droplet volume would be better controlled. The droplet would have to pool on top of the needle aperture before falling, and so it was possible to pull back on the plunger to prevent multiple droplets being released.

In these experiments 6 mixtures of water and glycerol (99\%, Sigma Aldrich Riedel-de Haen) solution $-0 \%, 20 \%, 40 \%, 60 \%, 80 \%$ and $100 \%$ glycerol solutions- were chosen (see Table 1 for properties). Droplets of each solution were released from various heights from 2 $\mathrm{cm}$ to $25 \mathrm{~cm}$ onto a loosely packed powder bed using a $100 \mu \mathrm{L}$ Hamilton syringe with a 22 gauge needle. In this set of experiments $2 \mathrm{~mL}$ of food dye (Queen Fine Foods Pty Ltd) was added to initial $10 \mathrm{~mL}$ glycerol-water mixture to increase contrast when taking photos. This affected the actual concentration of glycerol. We refer to the fluids by their names before adding the dye $(20 \%, 40 \%$, etc) but the true concentration (including the dye) has been given in the Table 1. The data shown in Table 1 has been interpolated from water-glycerol solution data, assuming that the dye has the same viscosity as water. This is a reasonable method to estimate the solution viscosity. The presence of the food dye reduced the surface tension of the fluids. Table 1 summarises the surface tensions of the fluids as measured using a dynamic 
surface tensiometer (Nima Technology, DST 9005) with a platinum DuNouy Ring (ring diameter $20.6 \mathrm{~mm}$ and wire diameter $500 \mu \mathrm{m})$.

The kinetic energy of the droplets was calculated from the potential energy of the droplet. By keeping the droplet volume constant at $0.02 \mathrm{~mL}$ and accounting for differences in fluid density, the kinetic energy can be varied by changing the release height of the droplet. We assume that the potential energy of the droplets is transformed completely into kinetic energy and this kinetic energy is used upon contact with the powder bed to deform the droplet such that coverage of the droplet is attained via internal flow of the droplet dragging particles onto it. Kinetic energy losses via powder packing rearrangement, including formation of a crater in the powder bed has not been taken into account.

The effect of powder size was investigated using a similar methodology. Four different size grades of PTFE powder (Sigma Aldrich Pty Ltd) - 100, 35, 12, and $1 \mu \mathrm{m}$ grades - were used to produce $1.2 \mathrm{~cm}$ high loosely packed powder beds with $61 \%, 75 \%, 78 \%$, and $87 \%$ porosity for $100,35,12$, and $1 \mu \mathrm{m}$ particle size, respectively. For these experiments, drops of a water solution $(10 \mathrm{~mL}$ distilled water plus $2 \mathrm{~mL}$ dye) were dispensed using the same $100 \mu \mathrm{L}$ syringe onto the powder beds, and the liquid marble images were analyzed as above.

\subsection{Image Analysis Method}

After the drop had landed, the fractionally covered liquid marbles were then photographed using a stereo microscope (SMZ series) with a $3 \mathrm{MP}$ camera at $1024 \times 768$ resolutions using Motic camera imaging software. Two images per droplet were taken from directly overhead: 
one with the upper surface of the droplet in focus to capture the fine details of the droplet (see Figure 2a) and the other with the outermost circumference of the droplet in focus. This latter image was used to trace the border of the droplet in NIH Image $\mathrm{J}$ software (V1.38X) so that the region of interest could be saved and applied to the former image.

After the two images were acquired, the image processing and analysis phase were performed. Firstly, the background was changed to black and foreground to white and the image was then segmented using "k-means clustering" (see Figure 2b). Reflections of the fiber optic lighting globes were manually edited out (compare white vs black spots in Figures $2 \mathrm{~b}$ and $2 \mathrm{c}$ ). The image was then converted to a threshold image with a setting of 0-5 (see Figure 2c) and the percentage coverage in the region of interest was calculated for images including fine details of gaps in the powder sheet (see Figure 2 a versus $2 \mathrm{c}$ ).

A fine crack in the powder sheet which we refer to as a "vein" was not a point of interest but affected the percentage coverage results. For this reason, the image processing was continued from the above step to eliminate the veins. The threshold image was converted to a binary image. The resulting image will have the covered region in black and the rest of the image in white (see Figure 2d). The image was then inverted and dilated twice and eroded once such that the uncovered vein like region diminished. If veins still exist they are either likely to be large enough to be important, or they are cut off from the main uncovered region and only present as small islands which can be omitted by the "particle analysis" tool in Image $\mathrm{J}$. Then, the percentage coverage in binary images was calculated using Image $\mathrm{J}$.

Five repetitions of kinetic energy experiment were made for each combination. Figure 2 shows the process of image $\mathrm{J}$ analysis for a mixture of $20 \%$ glycerol-water droplet which was 
released from $10 \mathrm{~cm}$ above a powder bed of PTFE $100 \mu \mathrm{m}$. The percentage of coverage for this system varied from $69.7 \%$ before deleting veins (Figure2c) to $81.4 \%$ after deleting veins (Figure 2d).

\section{Results and Discussion}

\subsection{Spreading coefficients theory.}

According to Rowe [5], the sign of the spreading coefficient $\lambda_{S L}$ can be used to predict whether the solid powder would spread on the liquid surface or not. The surface energies of all the powders and fluids used in these experiments are summarised in Table 2. Results for the calculated values of $\lambda_{S L}$ using equation (2) are tabulated in Table 3.

Rowe [5] proposed that spontaneous spreading of solid powder should occur when $\lambda_{S L}$ is positive. Table 3 shows that that $\lambda_{S L}>0$ for pure liquids on PTFE which means that spreading of solid powder over liquid should be spontaneous. On the other hand, all combinations of PTFE and pure liquids have $\lambda_{L S}<0$, which predicts that spreading of the liquid over the solid should be thermodynamically unfavorable. Predictions of powder behaviour based on pure liquids and PTFE are in contradiction with the experimental observations for drops placed gently on the powder bed from a height of $0 \mathrm{~cm}$. These drops show no powder coverage (Table 3). Since no consideration of external forces is given in the derivation of equations from (1) to (4), the zero release height experiment simulates the condition where the drop is under no external force. Thus, if liquid marble formation was solely a surface chemistry effect, with positive $\lambda_{S L}$ it would be expected that a liquid marble would spontaneously form. Therefore, 
we find that equation (2) proposed by Rowe [5] does not predict the spreading of powder over a liquid surface and therefore can not be used to predict liquid marble formation.

Apart from pure fluids, two polymer binder solutions were also studied for their interactions with powders. It is known that for polymer-water solutions the dispersive and polar components cannot be determined using equation (2) because of the possible adsorption of polymers on the surfaces of the polymer solutions. The spreading of powder over these polymer surfaces could not be experimentally observed. Literature surface tension data of PVP(6\%) and HPMC(2\%) solutions [9] show that these polymers quite significantly influence the surface tension of water (Table 2). PVP and HPMC reduce the polar component of water by $50.6 \%$ and $41.2 \%$, respectively, and change the dispersion component of water by $30.3 \%$ and $-15.6 \%$, respectively. Teflon powder shows no tendency of spreading on any of the liquids. The non-spreading behaviour of Teflon power over these polymer solutions further suggests that comparing surface free energy values of the liquid and solid phases (through $\lambda_{S L}$ ) is not a valid predictive indicator for solid powder spreading over liquid surface.

Figure 3 also shows variations in the amount of powder coverage when the drops were released from a $10 \mathrm{~cm}$ height. Although there is some variation in the amount of coverage due to local powder packing, the viewing angle, rolling on landing, etc., the amount of powder coverage appears to decrease as the fluid viscosity (provided in Table 2) increases. The viscosity effect is more systematically investigated in the following section 3.2

An obvious reason for the inability of $\lambda_{S L}$ to predict powder spreading over a liquid surface is that equation (2) does not correctly capture the physical process of powder spreading over a liquid surface. When hydrophobic powder spreads over a liquid surface, solid powder 
particles do not increase their surface area as they spread. Instead, powder aggregates merely disintegrate when they expand their coverage over the liquid surface [20]. In this process, inter-particle attraction forces must be overcome in order for powder particles to spread over a liquid surface. However, the inter-particle attraction forces cannot be equated to the work of cohesion of the solid surface [20]. Therefore $\lambda_{S L}$, which is a comparison of liquid-solid adhesion and solid "cohesion", does not reflect the physical process of powder spreading correctly. This is an area of ongoing research effort [20].

\subsection{Effect of kinetic energy on droplet coverage.}

An alternative mechanism for liquid marble formation is that powder motion around the shell is driven entirely by kinetic energy [2, 3]. Pilot scale studies of dry water formation showed that increased agitation promoted liquid marble formation [3]. Other studies have shown that an impinging drop undergoes flow circulation in the droplet interior and consequent surface flow was observed and modelled $[11,12]$. We believe this flow within the droplet is responsible for liquid marble formation [2], and that the driving force is the kinetic energy applied to the system $[2,3]$.

If the kinetic energy of drop impact on a solid surface is sufficiently high, the drop will first deform and flatten on impact, increasing the maximum radius of the droplet and thus increasing the area of contact between the solid surface and the drop. After the drop has deformed and flatten, the surface tension will cause the drop to recoil back towards a spherical shape. Both the deformation at impact and the recoil after impact create internal flow within the droplet interior, which drives a corresponding surface flow around the drop entraining particles $[11,12]$. When a fluid drop lands on a hydrophobic powder surface, it 
does not penetrate into the powder pores but "sees" an effectively solid powder surface [13]. As soon as the drop touches the powder, a small section of powder will adhere to the base of the drop. This is the initial step in forming the powder shell of a liquid marble. As the drop deforms and flattens, the contact area between the fluid and powder will increase, and more powder will adhere to the base of the drop. The overall surface area of the drop also increases since the drop is no longer spherical. This process of increasing contact area and increasing powder pickup continues until the maximum drop deformation is reached, and drop recoil commences. During drop recoil, the droplet returns to a spherical shape. The total surface area of the drop decreases back to its original spherical minima, and internal flow is created within the drop as the flattened shape recoils back to spherical. This internal flow creates a corresponding surface flow in the droplet, moving generally from the base of the drop towards the top. The combination of all of these processes - the increased powder-liquid contact area during deformation, the upward flow of fluid at the drop surface due to the recoils motion, and the temporary increase and then retraction of the drop surface area - all contribute to the formation of a liquid marble.

Since all these processes are enhanced by increasing the kinetic energy of impact, it follows that the degree of liquid marble powder coverage will be proportional to the kinetic energy of the drop as it lands on the hydrophobic powder surface. Although there is some evidence to support this hypothesis from preliminary results [2] and pilot scale studies [3], this hypothesis has not been rigorously tested.

\subsection{Effect of kinetic energy and fluid viscosity on droplet coverage.}

The first series of experiments focused on investigating the relationship between the kinetic energy imparted upon droplet and its coverage by powder on a flat loosely packed bed, and 
the second phase concentrated on particle size effect. Figure 3 shows the relationship between kinetic energy and percentage coverage for six water-glycerol solutions on $100 \mu \mathrm{m}$ PTFE powder. The X-scale error bars are representative of the maximum errors in height measurements $( \pm 0.5 \mathrm{~mm})$ and the subsequent variation in kinetic energy calculation. The Yscale error bars represent one standard error of the mean for 5 samples per droplet.

Figure 3 shows that increasing the kinetic energy causes an exponential increase in the powder coverage for water droplets on $100 \mu \mathrm{m}$ PTFE powder. Initially, the liquid marble coverage increases rapidly with each increment in the applied kinetic energy. However, as the coverage gets closer to $100 \%$, the rate of coverage slows and eventually appears to plateau at a maximum value between $85 \%-95 \%$ of complete coverage. Compared with the same data series for water, glycerol liquid marbles have a much lower coverage for a given kinetic energy. For example, powder coverage for a glycerol droplet is around 50\% less than for a water droplet the same size released from the same height of $10 \mathrm{~cm}$ (see Figure 3). The strongest glycerol solution used in these experiments had a viscosity 500 times the viscosity of water which significantly impairs the deformation and recoil of the drop upon impact. This consequently gives lower coverage because so much of the kinetic energy is dissipated by viscous forces. In addition, the higher concentration glycerol solutions also have lower surface tensions (refer to Table 1) which reduce the drop recoil forces [14, 15]. Subsequently, higher kinetic energy is needed to expand the drop-powder surface area at contact and also to produce good bulk fluid motion during deformation and recoil of the viscous drops. Thus, the percentage of coverage for glycerol droplet is much lower than for a water droplet at same given kinetic energy. $\mathrm{s}$ 
The liquid marble coverage data shown in Figure 3 is related to kinetic energy using the following empirical equation.

$$
\text { Coverage }(\%)=A\left(1-e^{-b E}\right)
$$

where the $A$ is the maximum extent of liquid marble coverage (\%), and the $b$ represents the ease of liquid marble formation (\%coverage/unit energy) and the $E$ is the kinetic energy of impact. High values of $b$ means that only a small increment in kinetic energy is required to produce a considerable increase in liquid marble coverage. The values of the parameters $\mathrm{A}$ and $\mathrm{b}$ were determined by fitting equation (5) to each data set shown in Figure 3 and minimising the sum square of errors. The results are summarised in Table 4, which shows that the maximum liquid marble coverage $A$ is a strong function of the fluid viscosity. Figure 4 plots the maximum coverage $\mathrm{A}$ as a function of the fluid viscosity, and shows the maximum coverage achieved falls sharply as the viscosity increases due to increasing fluid resistance to motion.

Figures $3 \& 4$ show that the higher the viscosity of the solution, the lower the degree of coverage. Table 4 also shows that the maximum extent of coverage A decreases as surface tension decreases due to the loss of driving force for drop recoil. Note that the surface tension of the fluids used varies over a narrow range (see Table1) compared to the several orders of magnitude variations in fluid viscosity. This again supports the conclusion that surface energy effects are not the main factor in determining the liquid marble powder coverage, as Figure 4 shows large differences in coverage where there are differences in viscosity but comparatively small changes in surface tension. The $20 \%, 40 \%$ and $60 \%$ glycerol data all overlap because the viscosity differences of these three solutions are relatively small (see Table 1 which shows 
viscosity varies between $1-5 \mathrm{mPa} . \mathrm{s})$. Fluids with higher viscosity and lower surface tension require higher kinetic energy input to produce the same liquid marble coverage.

The proposed model for liquid marble formation by kinetic energy involves deformation and an increase in surface area of the droplet upon impact followed by recoil which drives fluid flow. Thus we expect kinetic energy, surface energy and viscosity will all be important factors in determining liquid marble coverage. Plots of the coverage versus various combinations of dimensionless groups relevant to drop deformation and recoil $[13,15,16]$, including the modified Weber number, Ohnesorge number, Capillary number and Bond number did not produce an improved analysis or show a more general trend, and generally looked similar to Figures 3. The reason for the failure of dimensionless analysis in this case is not understood. The conventional analysis of drop impact required high speed dynamic imaging of the drops to determine the maximum spreading diameter, which can then be shown to be related to various functions of We and Re [e.g. 13, 15, 16]. We do not have this data available, and impact on the deformable powder bed creates a "crater" where the drop sinks below the top surface of the bed, which makes capturing the required images much more difficult compared to studying drop impacts on solid, immovable surfaces. Although we expect that the percentage coverage should be a function of the Weber and Reynolds numbers, our data does not support a simple overarching dimensionless relationship between liquid marble powder coverage and dimensionless groups.

\subsection{Effect of particle size on droplet coverage.}

The effect of particle size was investigated by repeating the experiments for different particle sizes of PTFE. Figure 5 shows that the same exponential relationship between kinetic energy and percentage of coverage was found, but the smaller particle sizes exhibit less 
coverage (see Table 5 and Figure 5). This is contrary to what is expected - a smaller particle is lighter and should be more easily carried by internal flows of the droplet. We believe that particle agglomeration confounded the effect of particle size. Aside from PTFE $100 \mu \mathrm{m}$, all the smaller particle size grades formed agglomerates which were clearly larger than the stated particle size and in some instances formed aggregates even greater than 100 microns. Figure 6 clearly demonstrates these agglomeration phenomena. These agglomerates hindered liquid marble formation as they resisted movement due to their higher mass. Thus our results show that the percentage of coverage increases as primary particle size of the powder increases, due to the lower level of agglomeration for the coarser powders tested.

Figure 6a clearly shows PTFE $1 \mu \mathrm{m}$ particles containing agglomerates much larger than the theoretical $1 \mu \mathrm{m}$ particle size. As shown in Figure 6d for PTFE $100 \mu \mathrm{m}$, all of the particles are similar in size and no agglomeration is observed. We expect the trend of increasing coverage for larger apparent particle size would be reversed for powders where the particles remained well dispersed, although agglomeration of fine particles is well known and extremely common. Kendall (1994) reported that cohesion forces (van der Waals force) for particle with $1 \mu \mathrm{m}$ size can be up to million times greater than gravity forces depending on the particle size and roughness of the surface in contact [19]. The strong cohesion forces between the finer particles would result in aggregation of the powders, as shown in Figure $6 a$, b, and may also increase the adhesion between the particles and the bulk of the powder bed. The inter-particle attraction between the particles in the bed would also resist the separation of aggregates and the formation of the powder shell around the liquid marble. More kinetic energy would be required to break the strong cohesion forces between particles as the primary particle size decreases, further retarding the extent of liquid marble coverage. 
The results in Figure 5 appear to show a critical height/kinetic energy where any additional kinetic energy input yields similar coverage. It may be that there is actually $100 \%$ coverage but due to minor image analysis bias $100 \%$ is not reported. Alternatively it may be that there is a limiting coverage amount $\mathrm{A}$ due to the powder properties, including powder packing causing gaps in the self-assembled powder layer, hydrophobic or static repulsion and limitations in level of droplet deformation that can be achieved.

Differences in particle packing within the petri dish may also have contributed to the unexpected trend in liquid marble coverage as a function of particle size. Often droplets impacting onto the powder bed cause the bed to deform and a "crater" is created. This reduces the overall amount of kinetic energy available for droplet coverage. As particle size decreases, the propensity for crater formation increases, due to the much lower bulk densities of the powder beds, which allow significant bed rearrangement and void collapse. Further investigation into the effects of particle size, aggregate size and powder bed structure is required to determine which is the most important effect.

In addition to the agglomeration issues already noted, the smaller particles were also less opaque compared to the larger $100 \mu \mathrm{m}$ PTFE powder. If the liquid marbles were only covered in a thin monolayer, the image analysis had more difficulty detecting the opacity (whiteness) of the particles, even if the liquid marble was fully covered. The small focal plane of the camera also aggravated this. Fine uncovered veins in the unfocused regions would be blurred and appear larger than they actually are and this dark areas left exposed would be counted as uncovered regions upon image analysis. This decreases the reported coverage from the true coverage, and this effect may have contributed to the measured 
maximum coverage and the parameter A being consistently less that $100 \%$, even when visual inspection of the liquid marbles appeared to show 100\% coverage. An example of this is shown in Figure 6c, where the side liquid marble can be seen to be well covered by a thin layer of powder, which is more difficult to see compared to the thicker layers on the top of the droplet.

\section{Conclusions}

By calculating solid-liquid and liquid-solid spreading coefficients for several liquid marble formulations confirmed that the spreading coefficient theory $[5,6]$ is inconsistent with experimental observations of liquid marble formation. An exponential relationship was found between increasing kinetic energy and the percentage of liquid marble coverage. The kinetic energy from impact causes an increase in drop surface area and the drop deformation and recoil create fluid flow which entrains the powder and forms the powder shell. By increasing the drop release height and therefore increasing kinetic energy, the liquid marble powder coverage increases, and the maximum extent of liquid marble coverage falls as viscosity increases and surface tension decreases. As particle size increased, higher coverage of the droplet was observed but these results were most likely confounded by the effects of agglomeration and/or powder bed rearrangement on impact. The results of this study are the first to study the formation mechanisms of liquid marbles and are an important step in understanding how to create liquid marbles as a precursor to a producing a wide range of structured powder-liquid products and advanced materials. 


\section{Acknowledgements.}

This work was financially supported by the Chemical Engineering Department and Faculty of Engineering at Monash University, and by the Australian Research Council Discovery Projects program (DP0770462). Scholarship support for N. Eshtiaghi was provided by the Monash Graduate Research School. Thanks to Leslie Yeo for initial discussions on kinetic energy effects.

\section{References:}

[1] P. Aussillous, D. Quere, Liquid marbles, Nature 411(2001) 924-927.

[2] K. P. Hapgood, B. Khanmohammadi, Granulation of hydrophobic powders, Powder Technology, special issue on granulation, 189 (2009), 253-262.

[3] L. Forny, K. Saleh, I. Pezron, P. Guigon, L. Komunjer, Influence of mixing characteristics for water encapsulation by self-assembling hydrophobic silica nanoparticles Powder Technology, special issue on granulation, 189 (2009), 263-269.

[4] K. P. Hapgood, L. Farber, JN. Michaels, Agglomeration of hydrophobic powders via solid spreading nucleation, Powder Technology, 188 (2009), 248-254.

[5] R. Rowe, Binder-substrate interactions in granulation, a theoretical approach based on surface free energy and polarity. International Journal of Pharmaceutics 52 (1989)149-154.

[6] S.Wu, Polar and non polar interactions in adhesion, Journal of Adhesion 5(1973) 39-55.

[7] D. R. Lide, Ed., CRC Handbook of Chemistry and Physics; CRC Press/Taylor and Francis: Boca Raton, 2008.

[8] P. Luner, M. Sandell, The wetting of cellulose and wood Hemicellulloses, Journal of Polymer Science.: Part C 28 (1969)115-142. 
[9] L. Zajic, G. Buckton, The use of surface energy values to predict optimum binder selection for granulations, International Journal of Pharmaceutics 59(1990)155-164.

[10] W. A. Zisman, Influence of constitution on adhesion, Industrial and Engineering Chemistry 55(10)(1963)19-39.

[11] L. Mahadevan, Y. Pomeau, Rolling droplets, Physics of Fluids 11(1999) 2449-2453.

[12] H. Fujimoto, N. J. Hatta, Deformation and rebounding processes of a water droplet impinging on a flat surface above leidenfrost temperature, Journal of Fluid Engineering 118(1996)142-149.

[13] D. Kannangara, H. Zhang, W. Shen, Liquid-paper interactions during liquid drop impact and recoil on paper surfaces, Colloids and Surfaces A: Physicochemical and Engineering Aspects 280(1-3) (2006) 203-215,

[14] S. Middleman, Modeling Axisymmetric Flows, Dynamics of Films, Jets, and Drops. San Diego, Academic Press, Inc., 1995.

[15] T. Mao, D.S. Kuhn, H. Tran, Spread and Rebound of liquid droplets upon impact on flat surfaces, AIChE 43(9) (1997) 2169-2179.

[16] H. Park, W.W. Carr, J. Zhu, J.F. Morris, Single drop impaction on a solid surface, AIChE Journal, 49(10) (2003) 2461-2471.

[17] I. Krycer, D.G. Pope, An Evaluation of Tablet Binding Agents. Part I. Solution Binders. Powder Technology, 1983. 34: p. 39-51.

[18] R.C. Rowe, Binder-substrate interactions in granulation: a theoretical approach based on surface free energy and polarity. International Journal of Pharmaceutics, 1989. 52: p. 149-154. [19] K. Kendall, Molecules and Mechanics, Science, 263(5154) (1994) 1720-1725.

[20] Nguyen, T.N., N. Eshtiaghi, K.P. Hapgood, W. Shen, An analysis of the thermodynamic conditions for solid powder particles spreading over liquid surface, submitted to Chemical Engineering Science, (2009). 
Table 1. Physical properties of fluids (including food dye) at $20^{\circ} \mathrm{C}$

\begin{tabular}{|l|c|c|c|}
\hline Fluid & $\begin{array}{c}\text { Actual composition } \\
\text { including food dye } \\
(\% \text { glycerol v/v) }\end{array}$ & $\begin{array}{c}\text { Surface tension } \\
(\mathbf{m N} / \mathbf{m})\end{array}$ & $\begin{array}{c}\text { Viscosity } \\
(\mathbf{m P a}) \text { ) }\end{array}$ \\
\hline Water & 0 & 71.4 & 1.0 \\
\hline Glycerol 20\% & 0.17 & 70.6 & 1.54 \\
\hline Glycerol 40\% & 0.33 & 69.2 & 2.68 \\
\hline Glycerol 60\% & 0.50 & 66.3 & 5.26 \\
\hline Glycerol 80\% & 0.67 & 64.6 & 9.8 \\
\hline Glycerol 100\% & 0.83 & 45.8 & 509 \\
\hline
\end{tabular}


Table 2: Summary of dispersive, polar and total surface energies.

\begin{tabular}{|c|c|c|c|c|}
\hline Powder or Fluid & $\begin{array}{c}\text { Dispersive } \\
\text { surface energy } \\
\left(\mathbf{m J} / \mathrm{m}^{2}\right)\end{array}$ & $\begin{array}{c}\text { Polar surface } \\
\text { energy } \\
\left(\mathbf{m J} / \mathbf{m}^{2}\right)\end{array}$ & $\begin{array}{l}\text { Total surface } \\
\text { energy }\left(\mathrm{mJ} / \mathrm{m}^{2}\right)\end{array}$ & $\begin{array}{c}\text { Fluid } \\
\text { viscosity } \\
\text { (mPa s) }\end{array}$ \\
\hline Water & $21.8^{[8]}$ & $51.0^{[8]}$ & 8] & $1.0^{[7]}$ \\
\hline Glycerol & $37.0^{[8]}$ & $26.4^{[8]}$ & $63.4^{[8]}$ & $509^{[7]}$ \\
\hline $6 \%$ PVP solution ${ }^{[9]}$ & 28.4 & 25.2 & 53.6 & 2.2 \\
\hline $2 \%$ HPMC solution ${ }^{[9]}$ & 18.4 & 30.0 & 48.4 & 3.1 \\
\hline PTFE [10] & 18.0 & 0.0 & 18.0 & -- \\
\hline
\end{tabular}


Table 3. Calculated spreading coefficients for several fluid droplets on $100 \mu \mathrm{m}$ PTFE (15X magnification, and $1200 \mu \mathrm{m}$ scale bar for all photos)

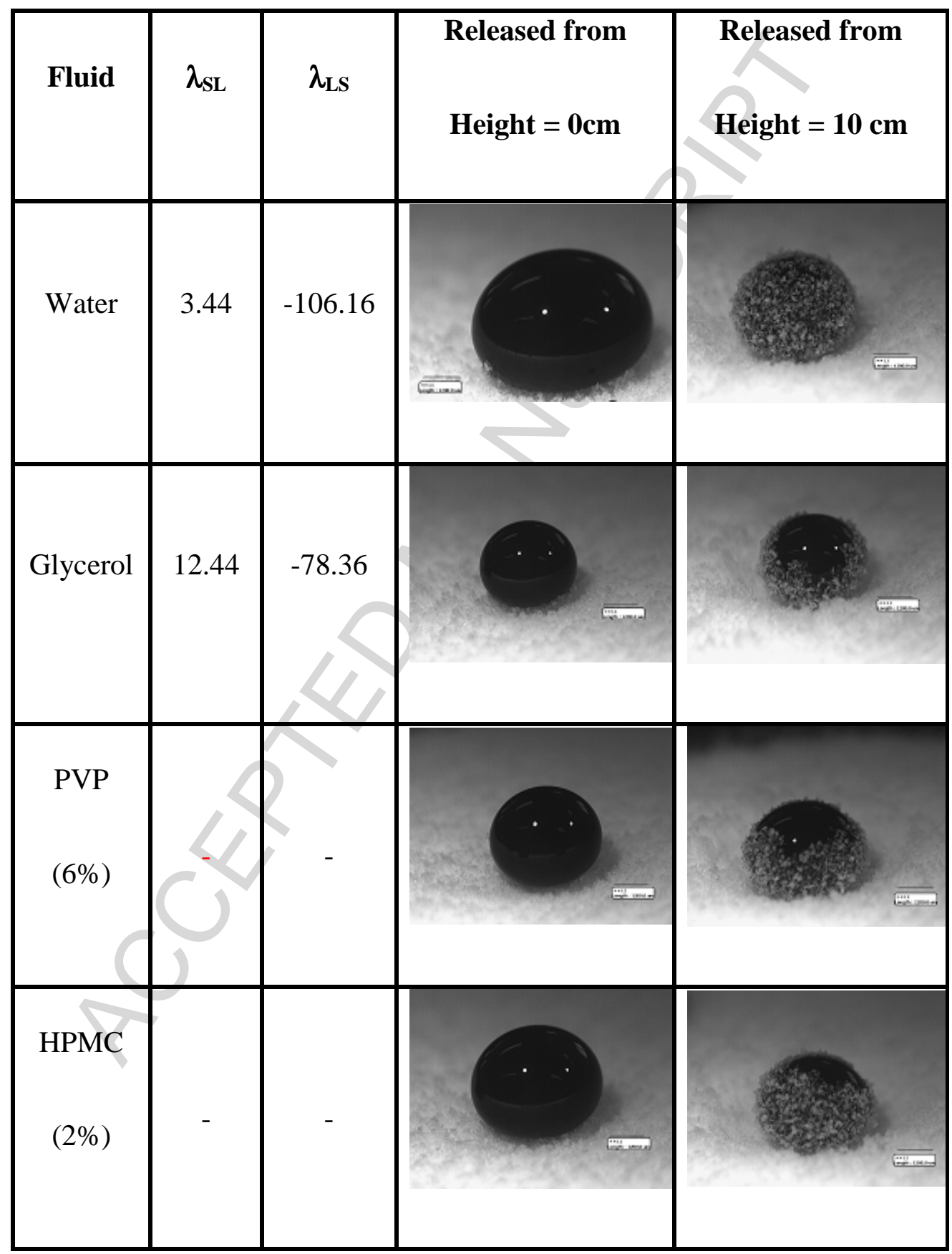


Table 4. Summary of the ease of formation, $b$, and maximum extent of coverage, A, for liquid marbles formed using $100 \mu \mathrm{m}$ PTFE powder

\begin{tabular}{|l|c|c|}
\hline \multirow{2}{*}{ Fluid } & Maximum & Ease of formation \\
& $\mathrm{A}(\%)$ & \\
\hline Water & 95 & 0.3 \\
\hline $20 \%$ Glycerol & 96 & $0.13 \mathrm{~J})$ \\
\hline $40 \%$ Glycerol & 96 & 0.12 \\
\hline $60 \%$ Glycerol & 94 & 0.13 \\
\hline $80 \%$ Glycerol & 93 & 0.1 \\
\hline $100 \%$ Glycerol & 84 & 0.045 \\
\hline
\end{tabular}

Table 5. The ease of formation and maximum coverage of water liquid marbles as a function of PTFE particle size.

\begin{tabular}{|c|c|c|}
\hline PTFE Particle & $\begin{array}{c}\text { Maximum } \\
\text { size }(\mu \mathrm{m})\end{array}$ & $\begin{array}{c}\text { Ease of } \\
\text { formation } \mathrm{b} \\
(\%)\end{array}$ \\
\hline 100 & 95 & 0.3 \\
\hline 35 & 96 & 0.3 \\
\hline 12 & 77 & 0.3 \\
\hline 1 & 77 & 0.4 \\
\hline
\end{tabular}




\section{Figures:}

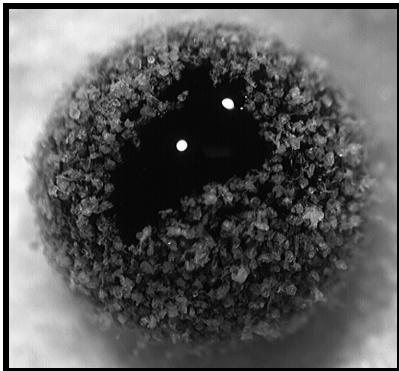

(a)

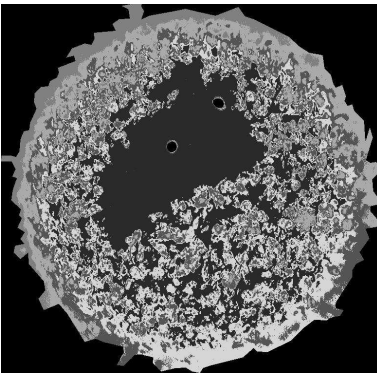

(b)

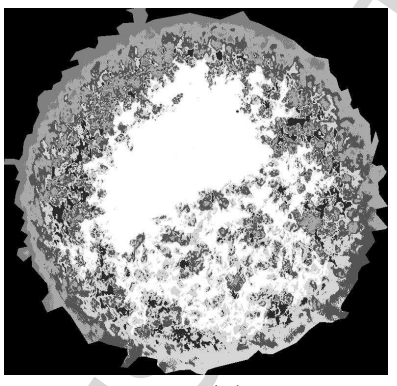

(c)

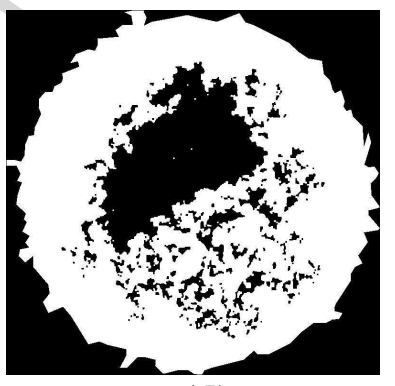

(d)

Figure 1. Image processing for $20 \%$ glycerol-water droplet on PTFE $100 \mu \mathrm{m}$ from $10 \mathrm{~cm}$

height: (a) original photo (b) clustered image after segmentation (c) after threshold (d) final image after binary processing

(a)
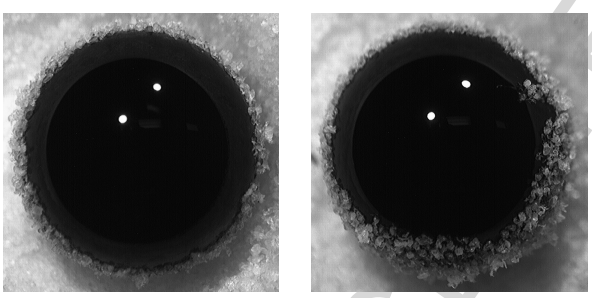

$10 \mathrm{~cm}$

$37 \%$ Cov.

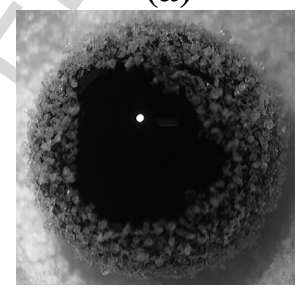

$15 \mathrm{~cm}$
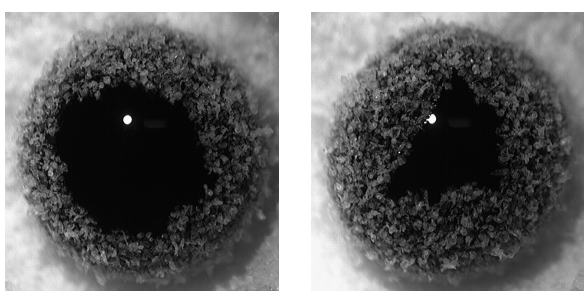

$5 \mathrm{~cm}$

$26 \%$ Cov.
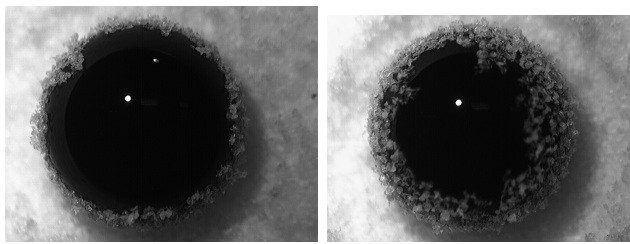

$4 \mathrm{~cm}$

$2 \mathrm{~cm}$

$26 \%$ Cov.

$62 \%$ Cov.

$20 \mathrm{~cm}$

$25 \mathrm{~cm}$

(b)

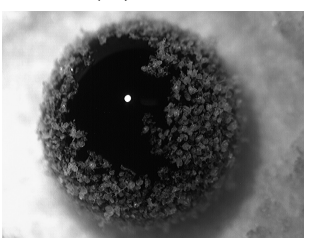

$6 \mathrm{~cm}$

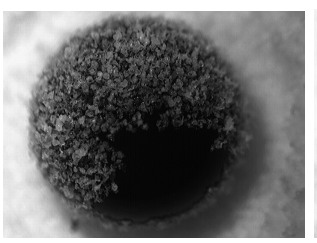

$8 \mathrm{~cm}$

81\% Cov. 73\% Cov.
$72 \%$ Cov.

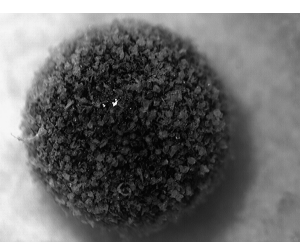

$10 \mathrm{~cm}$

Figure 2. Percent PTFE 100 powder coverage as a function of the drop release height for: (a) glycerol droplet (b) water droplet. 


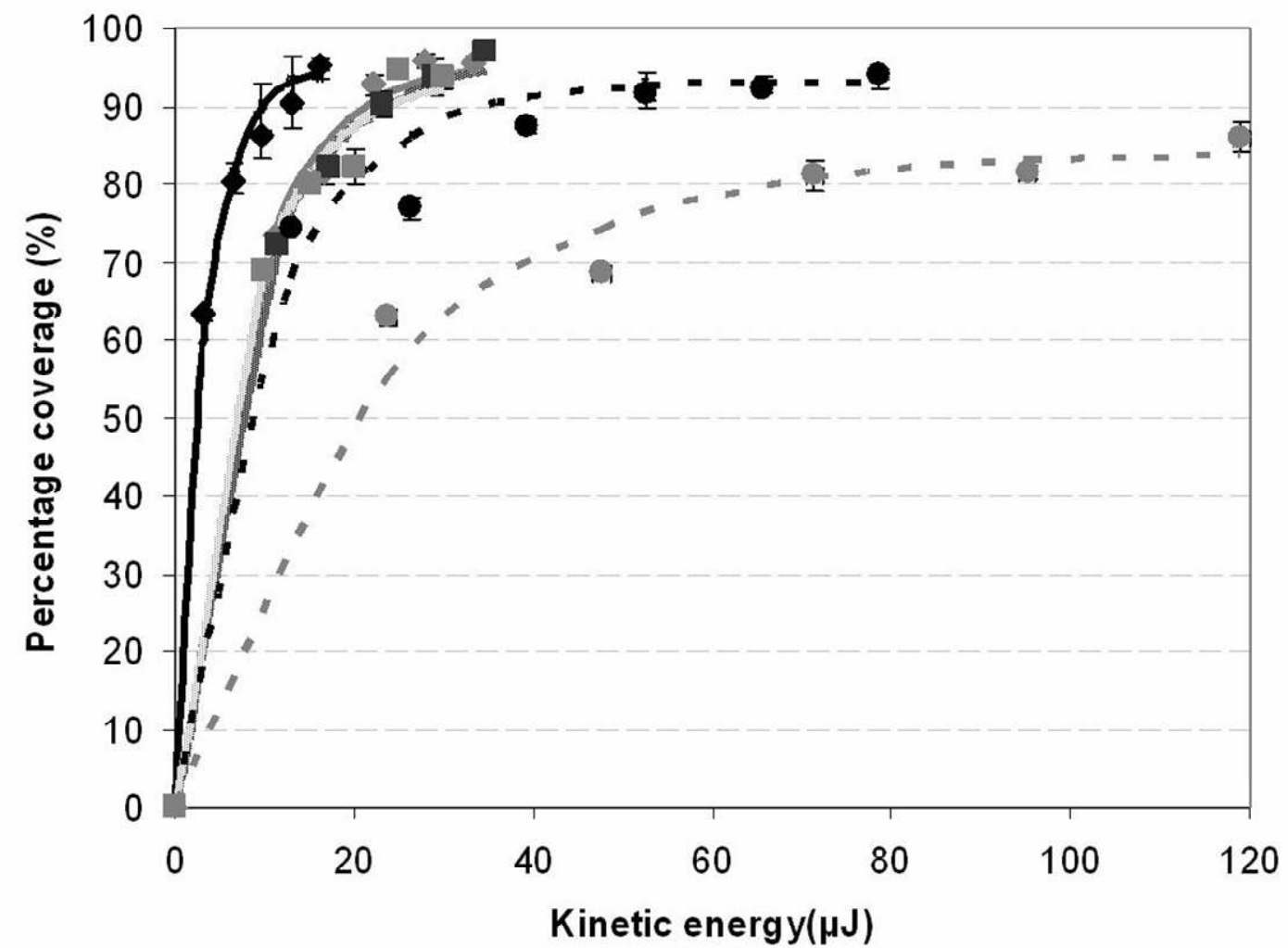

$\rightarrow-$ Water $\rightarrow-20 \%$ Glycerol $\rightarrow-40 \%$ Glycerol $-60 \%$ Glycerol $-\bullet-80 \%$ Glycerol - - Glycerol

Figure 3. Kinetic energy and viscosity effects on liquid marble powder coverage for water and glycerol solutions on $100 \mu \mathrm{m}$ PTFE powder 


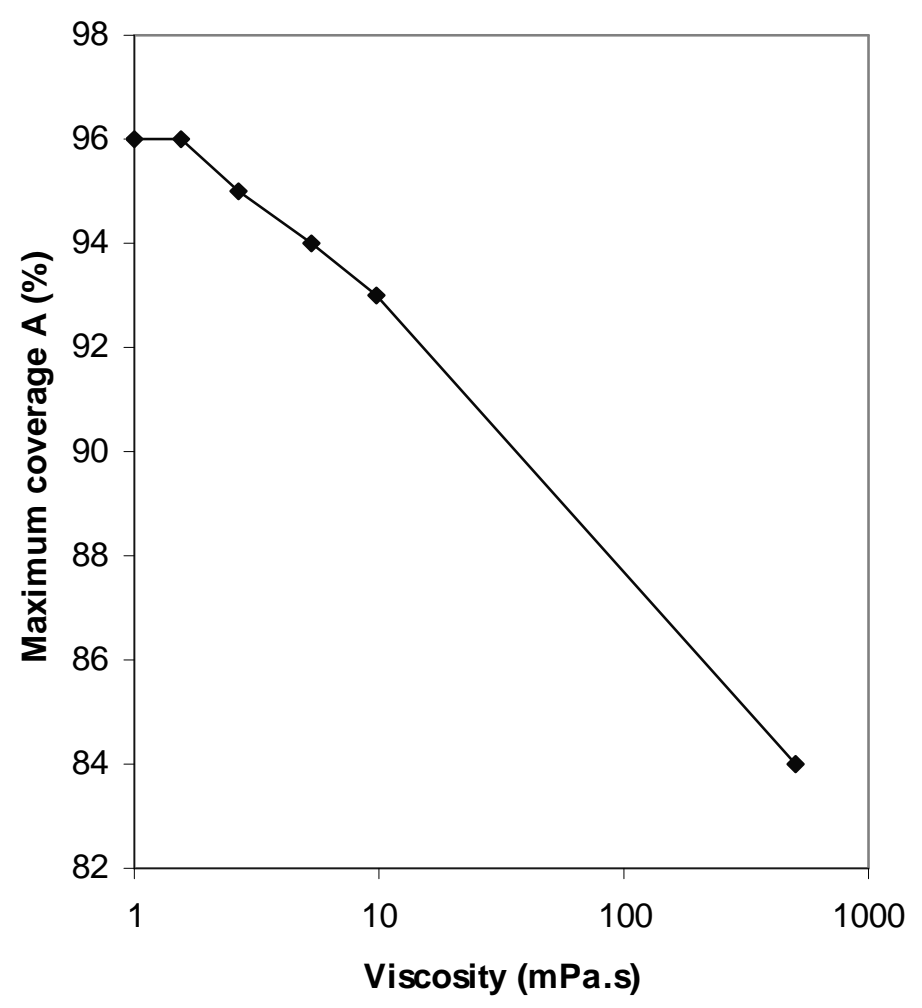

Figure 4 .The effect of fluid viscosity on maximum extent of coverage for water and glycerol solutions on $100 \mu \mathrm{m}$ PTFE powder 


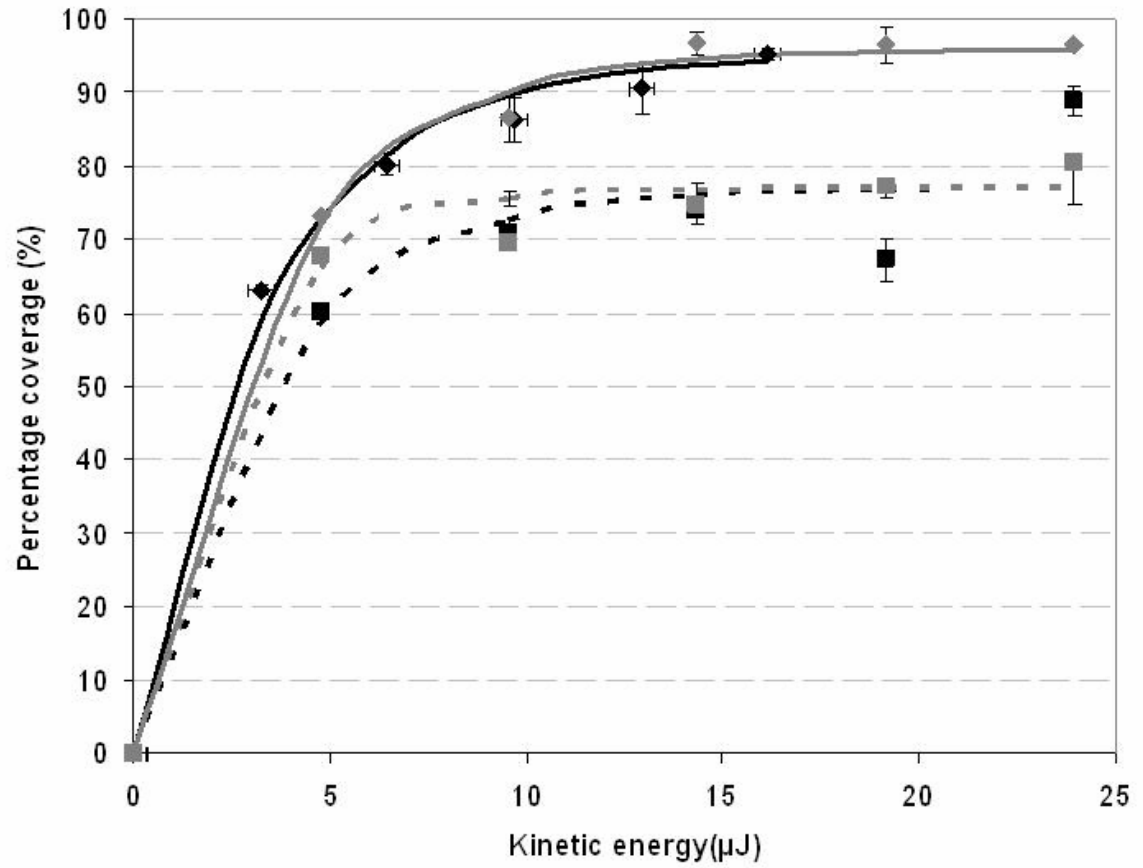

$\rightarrow$ PTFE $100 \rightarrow$ PTFE $35 \rightarrow$-PTFE $12 \rightarrow-$-PTFE 1

Figure 5. Particle size effect on liquid marble powder coverage for water droplets on PTFE powders. 


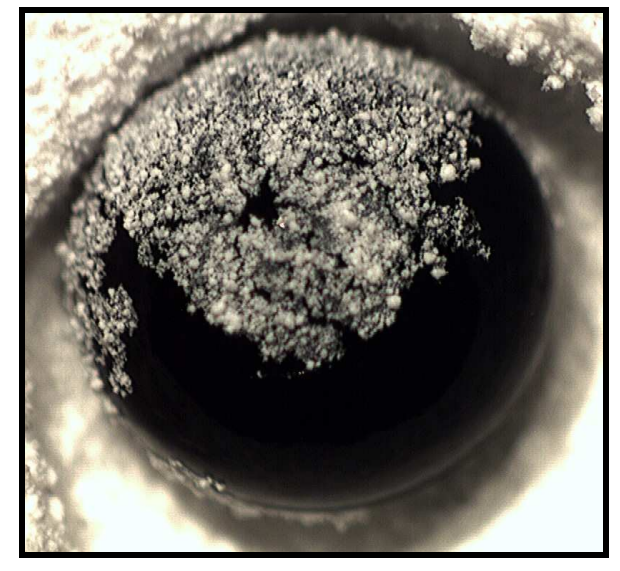

(a)

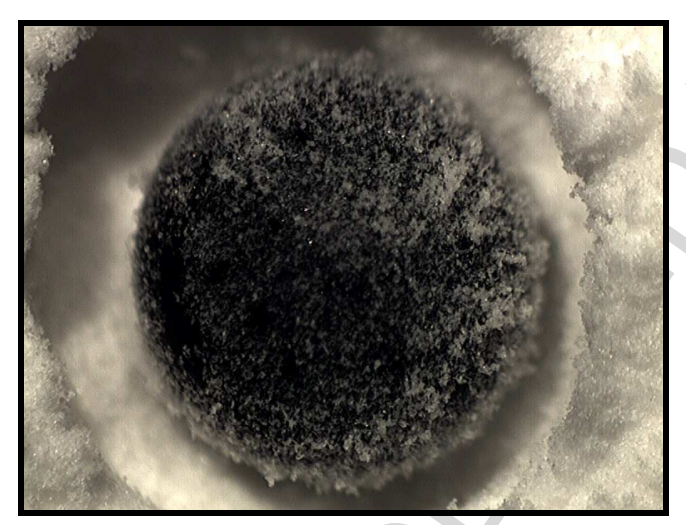

(c)

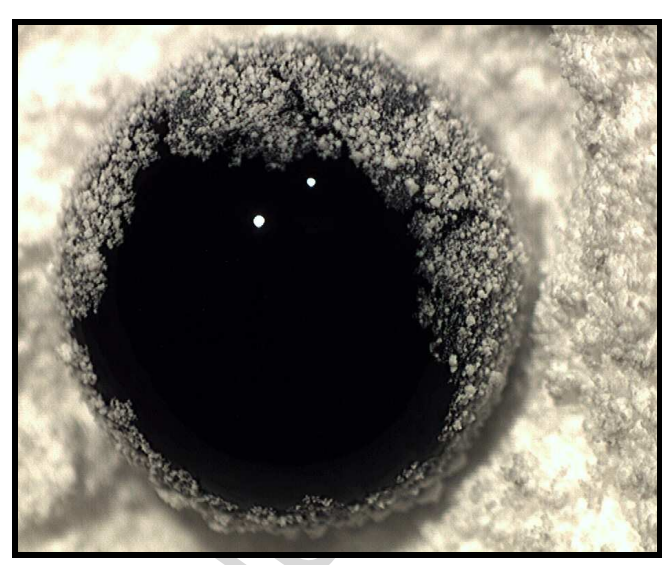

(b)

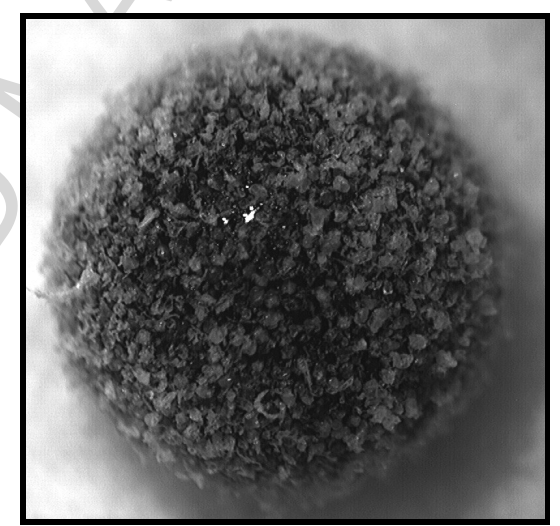

(d)

Figure 6. Water droplet released from 10cm height on (a) $1 \mu \mathrm{m}$ (b) $12 \mu \mathrm{m}$ (c) $35 \mu \mathrm{m}$ (d) 100 $\mu \mathrm{m}$ PTFE powder bed (droplet size and image magnification are constant) 